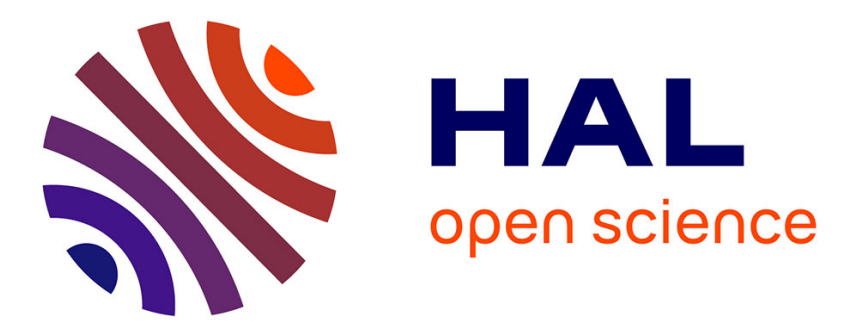

\title{
Revenue management in single-stage CONWIP production systems
}

Stratos Ioannidis, Vassilis S Kouikoglou

\section{To cite this version:}

Stratos Ioannidis, Vassilis S Kouikoglou. Revenue management in single-stage CONWIP production systems. International Journal of Production Research, 2008, 46 (22), pp.6513-6532. 10.1080/00207540701455343 . hal-00512991

\section{HAL Id: hal-00512991 \\ https://hal.science/hal-00512991}

Submitted on 1 Sep 2010

HAL is a multi-disciplinary open access archive for the deposit and dissemination of scientific research documents, whether they are published or not. The documents may come from teaching and research institutions in France or abroad, or from public or private research centers.
L'archive ouverte pluridisciplinaire HAL, est destinée au dépôt et à la diffusion de documents scientifiques de niveau recherche, publiés ou non, émanant des établissements d'enseignement et de recherche français ou étrangers, des laboratoires publics ou privés. 


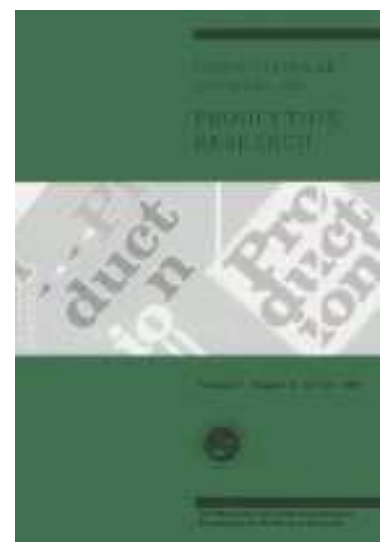

\section{Revenue management in single-stage CONWIP production systems}

\begin{tabular}{|c|c|}
\hline Journal: & International Journal of Production Research \\
\hline Manuscript ID: & TPRS-2006-IJPR-0963.R1 \\
\hline Manuscript Type: & Original Manuscript \\
\hline $\begin{array}{r}\text { Date Submitted by the } \\
\text { Author: }\end{array}$ & 21-Mar-2007 \\
\hline Complete List of Authors: & $\begin{array}{l}\text { Ioannidis, Stratos; University of the Aegean, Department of } \\
\text { Mathematics } \\
\text { Kouikoglou, Vassilis; Technical University of Crete, Dept of } \\
\text { Production Engineering and Management }\end{array}$ \\
\hline Keywords: & INVENTORY CONTROL, QUEUEING MODELS, PUSH/PULL SYSTEMS \\
\hline Keywords (user): & ADMISSION CONTROL, CONWIP \\
\hline
\end{tabular}

\section{scholarONE" \\ Manuscript Central}




\title{
Revenue management in single-stage CONWIP production systems
}

\author{
STRATOS IOANNIDIS $\uparrow$ and VASSILIS S. KOUIKOGLOU* $*$ \\ $†$ Department of Mathematics, University of the Aegean, 83200 Karlovassi, Samos, \\ Greece. \\ $\ddagger$ Department of Production Engineering and Management, Technical University of \\ Crete, University Campus, GR-73100 Chania, Greece. \\ *Corresponding author. Email: kouik@dpem.tuc.gr
}

\begin{abstract}
We study a joint admission/inventory control problem for a manufacturing system producing one product to meet random demand. The system employs a constant workin-process policy (CONWIP) whereby the total inventory of raw material and finished items is kept constant, and accepts orders only as long as the backlog is below a certain level. The objective is to determine the CONWIP and backlog levels that maximize the mean profit rate of the system. The system is modeled as a single server with a finite queue. It turns out that the mean profit rate is either concave or decreasing in one control parameter and also decreasing for large values of the other control parameter. A simple algorithm is developed which tracks down the globally optimum design in finite time. Numerical results show that the joint admission/inventory control policy achieves higher profit than other production control policies that have been examined in the literature.
\end{abstract}

Keywords: Production/inventory control; CONWIP systems; Admission control; Queueing systems. 


\section{Introduction}

Control of production systems addresses the questions of when to produce and when to accept an incoming order to maximize net profit, that is, revenue from sales less the costs of purchasing and processing raw items, carrying inventory, and backlogging customer orders. A practical approach to solve this problem is to find the best policy from a class of easily implementable policies that depend on a small number of parameters.

In this paper, we consider make-to-stock production systems, in which a production facility produces to satisfy a random demand for products of the same type. Among the inventory control policies used in multistage make-to-stock systems, CONWIP (Spearman et al. 1990) is perhaps the most attractive one, because it combines ease of implementation and satisfactory performance compared to other more complex inventory policies (see, e.g., Spearman and Zazanis 1992, Framinan et al. 2003 and the references therein). Under CONWIP, the system starts working with a fixed number of finished items and no raw items or orders pending. Thereafter, a raw item is released into the system only when a finished item is sold. Thus, the total inventory of the system is constant and is referred to as the CONWIP level. This policy ensures that the inventory cost is bounded.

If arriving orders cannot be filled immediately from stock, they are usually either rejected or accepted (see, e.g., Hadley and Whitin 1963, Smith 1977, Zipkin 2000). A strategy of accepted orders is known as the complete backordering policy (CB) whereas rejection of orders corresponds to a lost sales policy (LS). When the production rate is less than the demand rate, $\mathrm{CB}$ cannot be profitable since the number of outstanding orders would grow without bound. An alternative admission control policy is to always accept customer orders when stock is available and to accept or reject orders in a 
random manner (e.g. by performing a Bernoulli experiment) during stockouts independent of the current backlog. We then have a randomized admission control policy (RAC). RAC was proposed by Stidham (1985) for single-facility models and later by Porteus (1990, pp. 615-616) for the single-period newsvendor problem. Moinzadeh (1989) has derived expressions describing the operating characteristics of a single-stage production system under a combination of RAC and a base stock policy. It should be noted that LS and CB are special cases of RAC with admission probability zero or one, respectively.

Another order admission policy which also generalizes CB and LS is one that rejects orders when backlog reaches a certain limit and accepts them otherwise. This limit will be referred to as the base backlog and the corresponding policy as the base backlog policy (BB).

Policies combining CONWIP and BB have been studied in a number of papers for single-stage systems which do not incur costs for holding raw items. For such systems, CONWIP corresponds to a simple base-stock policy. Caldentey (2001) and Kouikoglou and Phillis (2002) have studied systems of the $M / M / 1$ type, that is, with exponentially distributed processing and interarrival times. They show that BB generalizes and outperforms CB and LS. Recently, Song (2006) has studied systems with an unreliable machine and exponentially distributed interarrival, processing, failure, and repair times. He shows the optimal policy a combination of a base-stock policy and a BB with two different base backlogs, which depend on the state of the machine (up or down). If the machine is completely reliable, then the optimal policy is the standard BB.

In this paper, we develop an algorithm to determine the optimal CONWIP level and base backlog for single-stage production systems that incur different costs for holding raw items and finished items. In Section 2, we model one such system as a single-server 
finite queueing system whose queue capacity $m$ is the sum of the CONWIP level and the base backlog. In particular, we examine systems for which the inventory/backlog level has a truncated geometric distribution except for two boundary states. A number of models, either exact or approximate, are presented for which the equilibrium probabilities have this form. In Section 3, we derive explicit expressions for the optimal CONWIP level for any fixed $m$. We also show that the value of $m$ that maximizes the mean profit rate of the system belongs to a bounded set. Using these results, we develop an algorithm which tracks down the globally optimum design in finite time. The numerical results of Section 4 show that BB outperforms LS, CB, and RAC.

\section{Model of a make-to-stock production system with partial backordering}

\subsection{System description}

Consider a production facility that produces a single product. Customers arrive at random times and each customer requests one unit of product. The times between successive customer arrivals are independent random variables with mean $1 / \lambda$. Processing times are also independent random variables with mean $1 / \mu$. Finished items are stored in an output buffer. An arriving order is satisfied immediately if there is at least one item in the output buffer; otherwise, the order is either backlogged or rejected.

The operation of the system is associated with four types of profit or cost:

$p$ unit profit (selling price less cost of purchasing raw material and processing per item),

$r$ unit raw material holding cost rate, which is the cost per unit time per item waiting to be or being processed in the system, 
$h$ unit holding cost rate, which is the cost per unit time per finished item held in the buffer,

$b$ unit backlog cost rate, which is the cost per unit time of delay of a pending order.

As discussed in Hadley and Whitin (1963, Section 1) and Porteus (1990), the holding cost rates $r$ and $h$ include the loss of opportunity of having capital invested rather than tied up in inventory as well as costs for renting and operating warehouses. Similarly, the unit backlog cost rate $b$ includes an opportunity cost due to delayed receipt of the revenue $p$ and, possibly, costs of estimating when the orders will be filled and notifying customers. In addition, $b$ may include the loss of customers' goodwill or a cost of the discount offered when an order takes too long to be filled. When an order is rejected, the lost sale results in a loss of the unit profit $p$ and a less tangible cost associated with the possible discouragement of the customer to place orders in the future. As we shall see in Section 3, this latter cost can be added to the unit profit in order to compute the mean profit rate of the system.

To avoid trivial cases, we assume that all the economic parameters defined above have positive values and that $p \mu$ is greater than $r$. Indeed, if $p \mu \leq r$, then the profit $p$ from selling one item would be no greater than the mean cost $r / \mu$ of holding one raw item over a production cycle and, therefore, the system should not operate.

Suppose that, initially, there are $s$ finished items and no raw items or orders pending in the production system. Thereafter, the system employs a CONWIP policy to control its inventory and a base-backlog policy to control its pending orders. Raw items are released into the system only when a finished item is sold, the facility stops producing when the output buffer contains $s$ items, and the system rejects an incoming order if the current number of backorders is $c$. Thus, $s$ is the CONWIP level and $c$ is the base 
backlog. This policy is attractive because it is easily implementable and optimal for $M / M / 1$-type systems with zero raw material holding costs, as discussed in the previous section.

The problem then is to find $s$ and $c$ that maximize the mean profit rate of the system in steady state. Next, we derive expressions for this quantity by analyzing an equivalent queueing system.

\subsection{Equivalent queueing system}

Consider a single-server queueing system with a finite capacity of $m=s+c$ customers. The times between successive customer arrivals and the service times are the same as the times between successive customer arrivals and the processing times in the production system. Let $n$ be the number of customers awaiting service in the queueing system, where $0 \leq n \leq m$. Suppose that, at time zero, there are $s$ finished items and no raw items or orders pending in the production system, and no customers in the queueing system, that is, $n=0$. Thereafter, as we shall see, whenever an event (order acceptance or production) takes place in the production system the same event (respectively, customer arrival or service completion) will occur in the queueing system. Therefore, these two systems will have equivalent sample paths.

Let $n_{b}$ the number of pending orders in the production system, $n_{r}$ the number of raw items, and $n_{h}$ the number of finished items. Note that, under CONWIP, the total inventory is constant; thus, we have $n_{r}+n_{h}=s$.

When $n \leq s$ in the queueing system, the production system has $n$ raw items, $s-n$ finished items, and no orders pending, that is, $n_{r}=n, n_{h}=s-n$, and $n_{b}=0$. When $n \geq s$, the production system is out of stock with $s$ raw items awaiting in front of the production facility and $n-s$ orders pending, that is, $n_{r}=s, n_{h}=0$, and $n_{b}=n-s$. In 
particular, when $n=0$, the queueing system is empty and its server idles. Then, in the production system, the output buffer is full, the production facility is stopped, and no pending orders are present. In a dual fashion, when $n=m$, the queueing system is full and all incoming arrivals are lost, whereas, in the production system, the output buffer is empty, $c=m-s$ orders are pending and all incoming orders are rejected.

Policies with a CONWIP level $s=0$ and base backlogs $c=m>0$ are special cases. By the standard definition of CONWIP, $s=n_{r}+n_{h}=0$ implies that the system does not operate because it has no raw items. Therefore, all policies having $s=0$ would become degenerate regardless of the choice of $m$, because they render the system inoperable just as the policy with $s=0$ and $m=0$. However, we can relax a little the constant work-inprocess requirement to broaden the class of candidate policies. Specifically, we assume that when $s=0$ and $m>0$, the system employs the, so-called, zero base-stock or maketo-order policy, whereby $n_{h}=0$ always whereas $n_{r}=0$ when there are no orders pending, otherwise $n_{r}=1$. In order to include the case $s=0$, we write $n_{r}=\max \{s, 1\}$ whenever $n_{b}>0$. On the contrary, the choice $s=1$ has the regular CONWIP interpretation, that is, $n_{r}+n_{h}=1$ with $n_{h} \in\{0,1\}$.

Table 1 relates the state variables $n_{r}, n_{h}$, and $n_{b}$ of the production system to the level $n$ of equivalent queueing system.

\section{INSERT Table 2 ABOUT HERE}

We assume that the production system has a steady state. The mean profit rate of the system equals the mean profit from sales less inventory and backlog costs. We shall compute these costs using the equilibrium probabilities $P_{n}$ of the equivalent queueing system, for $n=0,1, \ldots, m$. In general, these probabilities cannot be expressed in a closed form. However, there are models of production and queueing systems, either 
exact or approximate, for which $P_{n}$ has a simple algebraic form. In addition, our analysis requires that the equivalent queueing system becomes full or empty less frequently as $m$ increases, that is, $P_{0}$ and $P_{m}$ are decreasing functions of $m$. In the following two sections, we present these assumptions in detail.

\subsection{Assumption 1}

We assume that the stationary probabilities have a scalar geometric form except for certain boundary states. Specifically, we assume that

$$
P_{n}=\left\{\begin{array}{l}
K_{m} \alpha, \quad n=0 \\
K_{m} \sigma^{n}, \quad 1 \leq n \leq m-1 \\
K_{m} \sigma^{m} \delta, n=m
\end{array}\right.
$$

for $m \geq 2$, where $\sigma, \alpha$, and $\delta$ are positive parameters which depend on the statistics of the interarrival and service times but they are independent of $s, c$, and $m$. Finally, $K_{m}$ is a constant computed from the normalizing equation $\sum_{0}^{m} P_{n}=1$. Thus,

$$
\begin{aligned}
K_{m} & =\frac{1}{\alpha+\sigma+\ldots+\sigma^{m-1}+\sigma^{m} \delta} \\
& =\frac{1}{\alpha+\sigma \frac{1-\sigma^{m-1}}{1-\sigma}+\sigma^{m} \delta} \\
& =\frac{1}{A+D \sigma^{m}}
\end{aligned}
$$

where

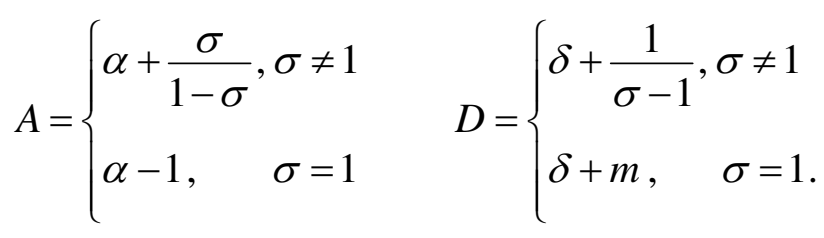

The parameters $\alpha$ and $\delta$ are associated with the two boundary probabilities $P_{0}$ and 
$P_{m}$. When $m=1$, the states 0 and $m$ are coupled and equation (1) may not hold. This case can be analyzed separately using exact or approximate Markovian models, which are computationally tractable because they involve a small number of states.

In the rest of the paper we assume that $\sigma \neq 1$. The results are also valid for $\sigma=1$, but their proofs require different, albeit simpler, algebraic manipulations and are omitted for brevity.

Equation (1) is applicable to a number of models, which we discuss next.

Model I: Setting $\alpha=\delta=1$ and $\sigma=\lambda / \mu$ in equation (1) we obtain the stationary probabilities of the $M / M / 1 / m$ queue.

Model II: Buzacott and Shanthikumar (1993) consider a discrete-time model of a transfer line with two unreliable machines having constant processing times and geometrically distributed times between failures and times-to-repair. In this model, all events of interest (production, failures, repairs) occur at times $1,2, \ldots$ Let $a_{i}$ and $b_{i}$ be the failure and repair probabilities respectively of machine $i$ in a production cycle, for $i=1,2$. Moreover, the failure probabilities of blocked or starved machines may differ from the nominal failure probabilities. Specifically, when the first machine is blocked, that is, $n=m$, its failure probability, denoted $c_{1}$, is $c_{1}=0$, if the machine does not fail during a blocking period (operation-dependent failures), or $c_{1}=a_{1}$, if the machine is vulnerable during a blocking cycle (time-dependent failures). In a dual fashion, when the second machine is starved we have $c_{2}=0$ or $c_{2}=a_{2}$. The stationary probabilities for this system are given in Section 6.6.1 of Buzacott and Shanthikumar 1993). We have shown that these have the form of equation (1) with

$$
\alpha=A_{2} B_{1} B_{2} \frac{1+\frac{A_{1}}{b_{1}}+\frac{c_{2} A_{1}+a_{2} B_{1}}{B_{2}}+\frac{\left[c_{2}\left(1-b_{1}\right)+a_{2} b_{1}\right] A_{1}}{b_{1} B_{2}}}{\left(A_{1}+B_{1}\right)\left(A_{2}+B_{2}\right)\left(c_{2} A_{1}+a_{2} B_{1}\right)},
$$




$$
\delta=A_{1} B_{1} B_{2} \frac{1+\frac{A_{2}}{b_{2}}+\frac{c_{1} A_{2}+a_{1} B_{2}}{B_{1}}+\frac{\left[c_{1}\left(1-b_{2}\right)+a_{1} b_{2}\right] A_{2}}{b_{2} B_{1}}}{\left(A_{1}+B_{1}\right)\left(A_{2}+B_{2}\right)\left(c_{1} A_{2}+a_{1} B_{2}\right)} \text {, and } \sigma=\frac{A_{2} B_{1}}{A_{1} B_{2}}
$$

where

$$
\begin{aligned}
& A_{1}=a_{1}+a_{2}-a_{1} a_{2}-a_{2} b_{1}, A_{2}=a_{1}+a_{2}-a_{1} a_{2}-a_{1} b_{2}, \\
& B_{1}=b_{1}+b_{2}-b_{1} b_{2}-a_{1} b_{2}, B_{2}=b_{1}+b_{2}-b_{1} b_{2}-a_{2} b_{1} .
\end{aligned}
$$

In our setting, the second machine represents the production facility. The first machine models an intermittent demand pattern that forms an alternating renewal process. Specifically, there are sequences of consecutive times during which customers place requests for one item per time unit followed by consecutive times during which no demand occurs. When $n=m$ the first machine (demand) is blocked, that is, all incoming orders are rejected but the demand pattern is not affected. Thus, for the first machine of this equivalent system, failures are time-dependent. Therefore, in the equations given above we must set $c_{1}=0$ and $c_{2}=b_{2}$.

Model III: Buzacott and Shanthikumar (1993) have also derived approximate expressions for the stationary probabilities of $G / G / 1 / m$ systems, that is, finite capacity queues with general interarrival and processing times, which have the form of (1) with

$$
\alpha=\frac{\sigma(1-\rho)}{\rho(1-\sigma)} \text { and } \delta=1
$$

Here, $\rho=\lambda / \mu$ and

$$
\sigma=\left\{\begin{array}{l}
\frac{N-\rho}{N}, \rho<1 \\
\frac{N^{\prime}}{N^{\prime}-\rho}, \rho>1,
\end{array}\right.
$$

where $N$ is an estimate for the mean number of customers in the $G / G / 1$ queue for $\lambda<\mu$, 
and $N^{\prime}$ is an estimate for the mean number of customers in the reversed $G / G / 1$ queue with arrival rate $\mu$ and service rate $\lambda$ when $\lambda>\mu$.

Model IV: Model III uses two parameters, $\alpha$ and $\sigma$. A richer approximation for $G / G / 1 / m$ systems was obtained by Kouvatsos (1986) by applying the principle of maximum entropy. His equations (2.9)-(2.11) involve three parameters, namely, $g, x$, and $y$, and are a special case of equation (1) with $\sigma=x, \alpha=1 / g$ and $\delta=y$.

\subsection{Assumption 2}

We assume that $P_{0}$ and $P_{m}$ are decreasing functions of $m$. This property has a number of implications, some of which are summarized in the following lemma.

Lemma 1. Suppose that $P_{0}$ and $P_{m}$ are decreasing functions of $m$. Then, for $\sigma \neq 1$, the following hold.

(a) For $A$ and $D$ given by equations (3), we have $A D<0$; specifically

$$
A(1-\sigma)>0 \text { and } D(\sigma-1)<0 \text {. }
$$

(b) The normalization constant is a decreasing function of $m$; specifically,

$$
K_{m}=K_{m-1}-K_{m-1} K_{m} \sigma^{m-1} D(\sigma-1)
$$

(c) The probabilities $P_{0}$ and $P_{m}$ are convex functions of $m$.

Proof. The proof is given in Appendix A.

Parts (a) and (b) of Lemma 1 will be used to prove certain structural properties of the mean profit rate of the system with respect to the CONWIP level and the base backlog. Part (c) of Lemma 1 provides additional insights on Assumption 2, whose validity is crucial in this article. Specifically, in most practical situations, as the capacity $m$ of a buffer increases, its state space increases and so then one expects that the 
probabilities of the states $n=0,1, \ldots, m$ will decrease. Also, in many production systems, the throughput rate appears to be a concave function of $m$ which implies that the probabilities of the buffer being full or empty are convex functions of $m$ (see, e.g., Buzacott and Shanthikumar 1993, pp. 183-186, 374-376).

In addition, we have verified Assumption 2 analytically for the four models presented in Section 2.3. In particular, for the two-machine system of Buzacott and Shanthikumar (1993), the parameters of $A$ and $D$ in the normalization equation (2) can be expressed as

$$
A=\frac{1}{1-\sigma} \frac{A_{2} B_{1}\left(a_{1}+b_{1}\right)\left(c_{2}+b_{2}\right)}{b_{1}\left(A_{1}+B_{1}\right)\left(c_{2} A_{1}+a_{2} B_{1}\right)}, D=\frac{1}{\sigma-1} \frac{A_{2} B_{1}\left(a_{2}+b_{2}\right)\left(c_{1}+b_{1}\right)}{b_{2}\left(A_{1}+B_{1}\right)\left(c_{1} A_{2}+a_{1} B_{2}\right)},
$$

from which it follows that conditions (4) are satisfied.

\section{Profit maximization}

In this section, we use the stationary probabilities (1) to express the mean profit rate as a function of the CONWIP level $s$ and the system capacity $m$, where $m=s+c$. Then, we derive expressions for the optimal CONWIP level for any fixed $m$. We also show that, under Assumption 2, the profit rate achieves its maximum value at some $m$ which belongs to a bounded set. This gives rise to an efficient algorithm for computing optimal values for $s$ and $m$.

Since in state $n=0$ the production facility is stopped, the average proportion of time the facility is busy is $1-P_{0}$. A busy period is defined as the interval between a startup and a stoppage of the production facility. Whenever the level of the output buffer drops from $s$ to $s-1$ the facility is switched on and a new production cycle begins. When a part is completed and the buffer level reaches the CONWIP level, the 
facility is stopped. From these observations, we see that each busy period of the facility contains an integer number of complete production cycles. These cycles are independent random variables with mean $1 / \mu$. Therefore, in steady state, the mean production rate of the system is $\mu\left(1-P_{0}\right)$. Since this quantity equals the mean arrival rate of accepted orders, the mean profit rate of the system is

$$
J(s, m)=p \mu\left(1-P_{0}\right)-r R-h H-b B
$$

for $m=0,1, \ldots$ and $s=0,1, \ldots, m$.

In some cases, there is a cost, say, $\beta$ per unit of lost sales in addition to the direct loss of sales margin $p$. This additional cost reflects the discouragement of customers to place orders in the future and it is a result of the loss of goodwill when their orders are rejected (see, e.g., Section 1.11 in Hadley and Whitin (1963) and Section 3.7 in Porteus (1990)). Then, the mean rate of lost sales is $\lambda-\mu\left(1-P_{0}\right)$ and the mean profit rate of the system is $J^{\prime}(s, m)=(p+\beta) \mu\left(1-P_{0}\right)-\lambda \beta-r R-h H-b B$. Since $\lambda \beta$ is independent of the control parameters, maximizing $J^{\prime}(s, m)$ is equivalent to maximizing $J(s, m)$ with $p+\beta$ in place of $p$.

Next, we derive equations involving the objective function $J(s, m)$ which we shall use to solve the optimization problem.

When the CONWIP level is positive, Table 1 and equation (1), which holds for $m \geq 2$, yield

$$
\begin{aligned}
R & =\sum_{n=0}^{s} n P_{n}+\max (s, 1) \sum_{n=s+1}^{m} P_{n} \\
& =K_{m}\left(1 \sigma+2 \sigma^{2}+\ldots+s \sigma^{s}\right)+K_{m} \max (s, 1)\left(\sigma^{s+1}+\sigma^{s+2}+\ldots+\sigma^{m} \delta\right)
\end{aligned}
$$




$$
\begin{gathered}
H=\sum_{n=0}^{s-1}(s-n) p_{n}=K_{m}\left[s \alpha+(s-1) \sigma+\ldots+1 \sigma^{s-1}\right] \\
B=\sum_{n=s+1}^{m}(n-s) p_{n}=K_{m}\left[1 \sigma^{s+1}+2 \sigma^{s+2}+\ldots+(m-s) \sigma^{m} \delta\right] .
\end{gathered}
$$

In the expressions given above and in the rest of this paper, all the sums involving geometric terms are defined appropriately or they become degenerate for extreme values of $s$ or $m$. For example,

$$
\begin{gathered}
1 \sigma+2 \sigma^{2}+\ldots+s \sigma^{s}=\left\{\begin{array}{cc}
0, & s=0 \\
1 \sigma+2 \sigma^{2}+\cdots+(m-1) \sigma^{m-1}+m \sigma^{m} \delta, & s=m
\end{array}\right. \\
\sigma^{s+1}+\sigma^{s+2}+\ldots+\sigma^{m} \delta=\left\{\begin{array}{cl}
\sigma^{m} \delta, & s=m-1 \\
0, & s=m .
\end{array}\right.
\end{gathered}
$$

For $s \geq 1$, the mean profit rate is computed from

$$
\begin{aligned}
J(s, m)=p \mu- & K_{m}\left\{p \mu \alpha+r\left[1 \sigma+2 \sigma^{2}+\ldots+s \sigma^{s}\right]+r s\left[\sigma^{s+1}+\sigma^{s+2}+\ldots+\sigma^{m} \delta\right]\right. \\
& \left.+h\left[s \alpha+(s-1) \sigma+\ldots+1 \sigma^{s-1}\right]+b\left[1 \sigma^{s+1}+2 \sigma^{s+2}+\ldots+(m-s) \sigma^{m} \delta\right]\right\}
\end{aligned}
$$

For the special case $s=0$, we have

$$
J(0, m)=p \mu-K_{m}\left\{p \mu \alpha+r\left[\sigma+\sigma^{2}+\ldots+\sigma^{m} \delta\right]+b\left[1 \sigma+2 \sigma^{2}+\ldots+m \sigma^{m} \delta\right]\right\}
$$

From the above equations, after a little algebra, we obtain

$$
J(s, m)=J(s-1, m)-K_{m}\left[h\left(\alpha+\sigma+\ldots+\sigma^{s-1}\right)+(r-b)\left(\sigma^{s}+\sigma^{s+1}+\ldots+\sigma^{m} \delta\right)\right]
$$

for $s=2, \ldots, m$ and

$$
J(1, m)=J(0, m)-K_{m}\left[h \alpha-b\left(\sigma+\sigma^{2}+\ldots+\sigma^{m} \delta\right)\right] .
$$

We now proceed to maximize $J(s, m)$. Since the problem is two-dimensional, we solve it sequentially. First, we maximize $J(s, m)$ with respect to $s$ for any fixed $m$ and then we track down the optimal value of $m$. 
For some fixed $m$, we seek a value $s_{m}$ for $s$ such that the optimality conditions

$$
\begin{gathered}
J(s, m) \geq J\left(s^{\prime}, m\right), \text { for every } s^{\prime} \text { such that } 0 \leq s^{\prime}<s \\
J(s, m)>J\left(s^{\prime}, m\right), \text { for every } s^{\prime} \text { such that } s^{\prime}>s
\end{gathered}
$$

hold simultaneously. The following theorem ensures that the maximizing CONWIP level $s_{m}$ can be tracked down recursively.

Theorem 1. If condition (4) holds, then either $s_{m}=s_{m-1}$ or $s_{m}=s_{m-1}+1$.

Proof. The proof is given in Appendix B.

We now turn to the problem of minimizing $J\left(s_{m}, m\right)$ with respect to $m$, where $m \in\{0,1, \ldots\}$. Clearly, when $m=0$ the system does not operate and, therefore, $s_{0}=0$ and $J(0,0)=0$. Also, as discussed Section 2.3, the case $m=1$ is not considered in this paper, but then one can develop a Markovian or even a simulation model to determine the optimal policy by comparing only two CONWIP levels since either $s_{1}=0$ or $s_{1}=1$. It then remains to maximize $J\left(s_{m}, m\right)$ on the set $\{2,3, \ldots\}$. If the maximum profit rate is less than or equal to zero, then the system is not profitable and we should not operate it. This holds trivially if $p \mu \leq r$, as discussed in Section 2.1. Hence, we assume that $p \mu>r$. The next result ensures that the optimal $m$ belongs to a finite set.

Theorem 2. If $p \mu>r$ and condition (4) holds, then there exist finite numbers $S$ and $M$ such that

(a) the system profitable, that is, $J(s, m)>0$, only if $s<S$;

(b) the optimal value for $m$ is bounded from above by $M$.

Proof. The proof is given in Appendix C.

Theorems 1 and 2 give rise to the following algorithm for tracking down the optimal control parameters. 


\section{Algorithm 1}

1) Compute the values $S$ and $M=\max \left\{M_{1}, M_{2}\right\}$ from equations (C1)-(C3) given in Appendix C. Set $m=0, s_{0}=0$, and initialize the globally optimal values $J=0$, $s=0, c=0$.

2) Increase $m$ one unit at a time. If $m=M$, then go to step 5, otherwise go to step 3.

3) Compute the profit rates for CONWIP levels $s_{m-1}$ and $s_{m-1}+1$. Let $J_{m}$ be the maximum profit rate and $s_{m}$ the corresponding optimal CONWIP level for the current $m$. If $J_{m}>J$, then update the globally optimal values, thus, $J=J_{m}, s=s_{m}$, and $c=m-s$.

4) If $s_{m} \geq S$, then go to step 5; otherwise go to step 2 .

5) The maximum profit rate is $J$, the optimal CONWIP level is $s$, and the optimal base backlog is $c$.

From a practical point of view, step 3 of Algorithm 1 employs equation (6) or (7) twice to compare the profit rates achieved by using CONWIP levels $s_{m-1}$ and $s_{m-1}+1$. This step is repeated at most $M$ times. For example, consider a production system with exponential interarrival and processing times, a mean annual demand of $\lambda=101$ items, a mean annual production capacity of $\mu=100$ items, and unit holding and backlog cost rates $r=h=b=0.01 p$, expressed as fractions of the unit profit $p$. For this system, we have $\alpha=\delta=1$ and $\sigma=\lambda / \mu=1.01$. From equations (C1)-(C3) we obtain $S=10000$, $M=M_{1}=10000$, and $M_{2}=9999$. In this case, an exhaustive search would require at most 10000 iterations of step 3 or about half a second on a Pentium® ${ }^{\circledR} 1.8 \mathrm{M}$ computer. Therefore Algorithm 1 is computationally efficient.

In the next section, we demonstrate that the proposed control policy achieves higher 
profit than other policies used in production systems.

\section{Numerical results}

In this section we compare the following six combinations of inventory and admission control policies:

BB: CONWIP/base-backlog

CB: CONWIP/complete backordering

LS: CONWIP/lost sales

RAC: CONWIP/randomized admission

MTO/BB: make-to-order (zero base-stock)/base-backlog

MTO/RAC: make-to-order/randomized admission

Let $(s, c)_{\mathrm{BB}}$ be a policy with a CONWIP level $s$ and a base backlog $c$. It turns out that $\mathrm{CB}$ is the same as $(s, \infty)_{\mathrm{BB}}, \mathrm{LS}$ is $(s, 0)_{\mathrm{BB}}$, and MTO/BB is $(0, c)_{\mathrm{BB}}$. Therefore one expects that $\mathrm{BB}$ will perform better than these policies. Let also $(s, q)_{\mathrm{RAC}}$ be a policy that uses a CONWIP level $s$ and accepts orders only with probability $q$, if these orders cannot be filled immediately from stock. Then, CB is the same as $(s, 1)_{\mathrm{RAC}}$, LS is $(s, 0)_{\mathrm{RAC}}$, and $\mathrm{MTO} / \mathrm{RAC}$ is $(0, q)_{\mathrm{RAC}}$.

We consider two production systems. The first one is equivalent to a finite $M / M / 1$ queue and the other is equivalent to Model II of Section 2.3. The standard parameters for the first system are $\lambda=9, \mu=10, r=h=b=5$, and $p=10$, and those of the second system are $a_{1}=0.4, b_{1}=0.6, a_{2}=0.2, b_{2}=0.4, r=h=b=5$, and $p=100$.

In Model II, the equivalent mean arrival and service rates are the efficiencies of the machines if they are operated in isolation. In this case, we have $\lambda=b_{1} /\left(a_{1}+b_{1}\right)$ and $\mu=b_{2} /\left(a_{2}+b_{2}\right)$. The parameters $\alpha, \delta$, and $\sigma$ are computed using the formulas given in 
Section 2.3 with $c_{1}=b_{1}$ and $c_{2}=0$, as discussed therein. Unfortunately Model II cannot be used directly to describe RAC, although it could be extended to take into account randomized admission. Thus, for simplicity, RAC was tested only for the $M / M / 1$ case by solving a simple birth-death Markov model. Specifically, when the number of customers in the equivalent queueing system is $n=s, s+1, \ldots$, the production system is out of stock, $n-s$ orders are pending, and the rate of additional customer orders accepted is $\lambda q$. When $n<s$, the system has $s-n$ finished items and all incoming orders are accepted. The mean profit rate of the system is a function of the CONWIP level $s$ and the acceptance probability $q$ during stockout periods. The optimal values for $s$ and $q$ are computed by exhaustive search.

We investigate the effects of varying $\lambda, b$, and $h$ on the mean profit rate, while fixing the other parameters to their standard values. Table 2 and figures 1-3 show the results for the $M / M / 1 / m$ system, and figures 4-6 show the results for Model II. The results of figure 4 are obtained by varying the parameter $b_{1}$ of Model II.

\section{INSERT Table 2 ABOUT HERE}

In Table 2, we observe that when $\lambda=3$, i.e., the demand rate is much smaller than the system's production capacity, the optimal CONWIP for BB and RAC is zero and all policies but LS achieve the same profit rate. In this case, it pays more to produce to order than producing to stock and this explains the poor performance of LS. When $\lambda$ is much greater than the production capacity $(\lambda=30)$, the optimal base backlog for BB decreases to $c=2$ and the admission probability for RAC is very small. The performance of LS is now very close to that of BB. For even larger values of $\lambda$, BB and RAC reject all incoming orders during stockouts and so they both become lost sales policies. 


\section{INSERT Figures 1-6, IN THAT ORDER, ABOUT HERE}

From these numerical results, we see that $\mathrm{BB}$ achieves the highest profit rates in all cases. For LS, CB, and MTO/BB this result agrees with intuition since these policies are special cases of BB. When the demand rate $\lambda$ is low, the profit rate of LS degrades, and when $\lambda$ is greater than or equal to $\mu, \mathrm{CB}$ is not profitable since the mean backlog of the system increases without bound. Also as the unit inventory cost $h$ increases or the backlog cost $b$ decreases, MTO/BB becomes less profitable than BB. However, as $\lambda$ tends to zero, the optimal base backlog under $\mathrm{BB}$ increases and so $\mathrm{CB}$ performs equally well as $\mathrm{BB}$. CB is also optimal when $b$ tends to zero, since backlogging the demand during stockouts increases the throughput of the system with no cost. In a dual fashion, as $\lambda$ or $b$ increases, backlogging is either unnecessary or costly and, therefore, the optimal base backlog decreases until BB becomes a lost sales policy.

Finally, from figures 1-3 and Table 2, we see that BB and RAC achieve about the same profit when $\lambda \rightarrow 0$ and when $b$ is either very high or very low. In the remaining cases BB provides a profit increase of $10-20 \%$ over RAC. However, in all cases, RAC is more profitable than $\mathrm{LS}, \mathrm{CB}$, and MTO/RAC since these policies are special cases of RAC. The superiority of $\mathrm{BB}$ over RAC is due to the fact that $\mathrm{BB}$, unlike RAC, is a state-dependent policy. Under RAC, an order is accepted or rejected with the same probability regardless of the backlog, so sales may be lost when only a few orders are pending and orders can be accepted while the backlog is high.

The advantage of BB over the other policies is that it balances inventories, backlog, and lost sales in an optimal fashion, and can adapt to seasonal demand fluctuations as well as to changes of the selling price and the unit inventory and backordering costs. 


\section{Conclusions}

We have studied threshold-type policies of admission and inventory control for a manufacturing system producing one product to satisfy demand. When no orders are present, the system produces until stock reaches a specified level, the CONWIP level. When stock is not available, the system accepts orders only when the number of pending orders is less than a specified level, the base backlog. As discussed in Section 1, this control policy is optimal for a class of single-stage production systems.

In this article, we have developed a computationally efficient algorithm to determine the optimal values for the CONWIP level and the base backlog. Sufficient conditions are provided under which this algorithm requires finite and, in fact, minimal CPU times to track down the optimal control parameters. These conditions are satisfied by the finite $M / M / 1$ queue and also by three more complex models which have been used in the past to model production systems. The numerical results show that the proposed policy achieves higher profit than other policies that have been examined in the production systems literature.

The idea of coordinating admission and inventory decisions could be applied to production systems with several demand classes and setup times. For systems that incur a setup cost or setup delay during a startup of the production facility, a practical control policy would be to combine an $(s, S)$ inventory policy (Iglehart 1963, Sethi and Cheng 1997) and the proposed partial backordering strategy. In an $(s, S)$ inventory policy, the production facility starts when the level of the output buffer drops to $s$ and stops when the level reaches $S$. Ha (1997a, b) has studied the problem of stock rationing when there are several demand classes, assuming that the backlog is either uncontrollable (complete backordering) or zero (lost sales). These two cases are special cases of the partial backordering policy which we have studied here. Future research will focus on such 


\section{Aknowledgements}

This work was supported in part by a research grant 'Herakleitos' of the Operational Programme for Education and Initial Vocational Training from the Greek Ministry of National Education and Religious Affairs.

\section{Appendix A. Proof of Lemma 1}

Since $P_{0}$ is decreasing in $m$, equation (1) yields $K_{m-1} \alpha>K_{m} \alpha$. Hence, $K_{m}$ is decreasing in $m$. Moreover, substituting equation (2) for $K_{m-1}$ and $K_{m}$ into the inequality $K_{m-1}>K_{m}$ yields

$$
\frac{\alpha}{A+D \sigma^{m-1}}>\frac{\alpha}{A+D \sigma^{m}}
$$

or, equivalently, $A+D \sigma^{m}>A+D \sigma^{m-1}$, from which we obtain the second inequality of condition (4). The first inequality of (4) follows similarly from the assumption that $P_{m}$ is 
a decreasing function of $m$. Therefore, $A$ and $D$ have opposite signs and are nonzero for $\sigma \neq 1$. Also, from

$$
\frac{K_{m}}{K_{m-1}}=\frac{A+D \sigma^{m-1}}{A+D \sigma^{m}}=\frac{A+D \sigma^{m}-\sigma^{m-1} D(\sigma-1)}{A+D \sigma^{m}}=1-K_{m} \sigma^{m-1} D(\sigma-1)
$$

we obtain equation (5). Finally, the convexity of $P_{0}$ follows from the fact that inequality

$$
2 \frac{\alpha}{A+D \sigma^{m}}<\frac{\alpha}{A+D \sigma^{m-1}}+\frac{\alpha}{A+D \sigma^{m+1}}
$$

is equivalent to $A D<D^{2}$, and the convexity of $P_{m}$ follows similarly from the dual inequality $A D<A^{2}$. These inequalities are true since $A D<0$.

\section{Appendix B. Proof of Theorem 1}

We examine two cases, namely, $r>b$ and $r \leq b$.

Case 1: $r>b$. From equation (8), we have

$$
\begin{aligned}
J(s, m-1)= & J(s-1, m-1) \\
& -K_{m-1}\left[h\left(\alpha+\sigma+\ldots+\sigma^{s-1}\right)+(r-b)\left(\sigma^{s}+\sigma^{s+1}+\ldots+\sigma^{m-1} \delta\right)\right]
\end{aligned}
$$

for $s \geq 2$. Since the term $(r-b)$ is positive, it follows that $J(s, m-1)<J(1, m-1)$ for all $s \geq 2$. Hence $s_{m-1} \in\{0,1\}$ and, by the same reasoning, $s_{m} \in\{0,1\}$. If $s_{m-1}=0$, then the theorem holds true since either $s_{m}=0$ or $s_{m}=1$. If $s_{m-1}=1$, then $J(1, m-1) \geq$ $J(0, m-1)$ and, using equation (9),

$$
h \alpha-b\left(\sigma+\sigma^{2}+\ldots+\sigma^{m-1} \delta\right) \leq 0
$$

The term $\left(\sigma+\sigma^{2}+\ldots+\sigma^{m-1} \delta\right)$ can be increased by adding the positive quantity $D(\sigma-1) \sigma^{m-1}$. Hence, we obtain the stronger inequality 


$$
h \alpha-b\left(\sigma+\sigma^{2}+\ldots+\sigma^{m} \delta\right)<0
$$

which, by equation (9), implies that $J(1, m)>J(0, m)$ and, therefore, $s_{m}=s_{m-1}=1$.

Case 2: $r \leq b$. The function $J(s, m)$ is concave in $s$ since, by equations (8) and (9),

$$
\begin{gathered}
{[J(s+1, m)-J(s, m)]-[J(s, m)-J(s-1, m)]=K_{m}(r-b-h) \sigma^{s} \leq 0, \text { for } s \geq 2} \\
{[J(2, m)-J(1, m)]-[J(1, m)-J(0, m)]=-K_{m}\left[h \sigma+b \sigma+r\left(\sigma^{2}+\sigma^{3}+\ldots+\sigma^{m} \delta\right)\right]<0 .}
\end{gathered}
$$

If $s_{m-1}=0$, then we have $J(0, m-1)>J(1, m-1)$ which, in view of equation (9), becomes

$$
h \alpha-b\left(\sigma+\sigma^{2}+\ldots+\sigma^{m-1} \delta\right)>0
$$

or, equivalently,

$$
h \alpha \sigma-b\left(\sigma^{2}+\sigma^{3}+\ldots+\sigma^{m} \delta\right)>0
$$

By Assumption 2, we have that $\alpha \sigma<\alpha \sigma+A(1-\sigma)=\alpha+\sigma$. Therefore,

$$
h(\alpha+\sigma)-b\left(\sigma^{2}+\sigma^{3}+\ldots+\sigma^{m} \delta\right)>0
$$

from which we deduce that

$$
J(2, m)-J(1, m)=-K_{m}\left[h(\alpha+\sigma)+(r-b)\left(\sigma^{2}+\sigma^{3}+\ldots+\sigma^{m} \delta\right)\right]<0 .
$$

Hence, $J(s, m)$ is decreasing at $s=2$ and also decreasing for all $s \geq 2$ because it is a concave function of $s$. Therefore, in this case, we have $s_{m}=0$ or 1 and the theorem holds. Next, we examine the case when $s_{m-1}=1$. The corresponding optimality conditions, by the concavity property we have established, are equivalent to the following inequalities:

$$
J(1, m-1)-J(0, m-1)=K_{m-1}\left[-h \alpha+b\left(\sigma+\sigma^{2}+\ldots+\sigma^{m-1} \delta\right)\right] \geq 0
$$

and 


$$
J(2, m-1)-J(1, m-1)=K_{m-1}\left[-h(\alpha+\sigma)-(r-b)\left(\sigma^{2}+\sigma^{3}+\ldots+\sigma^{m-1} \delta\right)\right]<0 .
$$

In the expressions given above, the equalities result from equations (8) and (9). The first inequality can be strengthened by adding the term $K_{m-1} b D(\sigma-1) \sigma^{m-1}$ to its left side. This yields $J(1, m)>J(0, m)$, or $s_{m} \geq 1$. Multiplying the second inequality by $\sigma$, we obtain

$$
h\left(\alpha \sigma+\sigma^{2}\right)+(r-b)\left(\sigma^{3}+\sigma^{4}+\ldots+\sigma^{m} \delta\right)>0,
$$

which can be strengthened by adding the term $h A(1-\sigma)=h(\alpha-\alpha \sigma+\sigma)$ to its left side to yield

$$
h\left(\alpha+\sigma+\sigma^{2}\right)+(r-b)\left(\sigma^{3}+\sigma^{4}+\ldots+\sigma^{m} \delta\right)>0 .
$$

By equation (8), this implies $J(2, m)>J(3, m)$. Hence $s_{m} \leq 2$. Therefore, $s_{m}=1$ or $s_{m}=2$. Finally, for $s_{m-1}=s \geq 2$, it can similarly be shown that the inequalities $J(s, m-1)-J(s-1, m-1) \geq 0$ and $J(s+1, m-1)-J(s, m-1)<0$ imply that either $s_{m}=s$ or $s_{m}=s+1$.

\section{Appendix C. Proof of Theorem 2}

To prove part (a) it suffices to examine the cases when $s_{m} \geq 1$, since otherwise we have $s_{m}=0$ and, thus, we can set $S=1$. Let $s=s_{m} \geq 1$. From equation (6) we have

$$
\begin{aligned}
J(s, m)= & p \mu-K_{m}\left\{p \mu \alpha+r\left[1 \sigma+2 \sigma^{2}+\ldots+s \sigma^{s}\right]+r s\left[\sigma^{s+1}+\sigma^{s+2}+\ldots+\sigma^{m} \delta\right]\right. \\
& \left.+h\left[s \alpha+(s-1) \sigma+\ldots+1 \sigma^{s-1}\right]+b\left[1 \sigma^{s+1}+2 \sigma^{s+2}+\ldots+(m-s) \sigma^{m} \delta\right]\right\} \\
\leq & p \mu-K_{m}\left\{p \mu \alpha+s \min (r, h) / K_{m}+b\left[1 \sigma^{s+1}+2 \sigma^{s+2}+\ldots+(m-s) \sigma^{m} \delta\right]\right\} \\
< & p \mu-K_{m} p \mu \alpha-s \min (r, h) .
\end{aligned}
$$


It follows from the above and the fact that $J(s, m)>0$ that $s<p \mu\left(1-K_{m} \alpha\right) / \min (r, h)$. Since the normalization constant $K_{m}=\left(A+D \sigma^{m}\right)^{-1}$ is decreasing in $m$, we have $K_{m}>K_{\infty}$. Moreover, $K_{\infty}=0$ if $\sigma>1$ and $K_{\infty}=1 / A$ if $\sigma<1$. Therefore

$$
s< \begin{cases}\frac{p \mu}{\min (r, h)}, & \sigma>1 \\ \frac{p \mu}{\min (r, h)} \frac{A-\alpha}{A}, & \sigma<1 .\end{cases}
$$

The second of the bounds given above is positive since $A=\alpha+\sigma /(1-\sigma)>\alpha$ when $\sigma<1$. A universal bound for $s$ is given by

$$
s<S=; \frac{p \mu}{\min (r, h)}
$$

Note that $p \mu>r$ implies $p \mu>\min (r, h)$. Hence, $S>1$. Note that equation $(\mathrm{C} 1)$ holds for all values of $\sigma$, including $\sigma=1$.

To prove part (b) of Theorem 2 we assume that $m$ is the optimal value of the control parameter. Hence, $J\left(s_{m}, m\right)$ is no less than $J(s, m-1)$ for any choice of $s$. We examine three cases, namely, $s_{m}=0,1 \leq s_{m} \leq m-1$, and $s_{m}=m$.

If $s_{m}=0$, then, by equation (7),

$$
\begin{array}{r}
J(0, m)-J(0, m-1)=-K_{m}\left\{p \mu \alpha+r\left[\sigma+\sigma^{2}+\ldots+\sigma^{m} \delta\right]+b\left[1 \sigma+2 \sigma^{2}+\ldots+m \sigma^{m} \delta\right]\right\} \\
+K_{m-1}\left\{p \mu \alpha+r\left[\sigma+\sigma^{2}+\ldots+\sigma^{m-1} \delta\right]+b\left[1 \sigma+2 \sigma^{2}+\ldots+(m-1) \sigma^{m-1} \delta\right]\right\} .
\end{array}
$$

From equation (5) of Lemma 1, after a little algebra, we obtain

$$
J(0, m)-J(0, m-1)=K_{m-1} \sigma^{m-1}\{[p \mu-J(0, m)-r-b(m-1)] D(\sigma-1)-b \sigma \delta\} .
$$

The above expression is nonnegative, since $s_{m}=0$ and we have assumed that $J\left(s_{m}, m\right) \geq$ $J(s, m-1)$ for all $s$. Moreover, the term $J(0, m)$ inside the brackets must be positive. 
From these observations we obtain

$$
m<M_{0}=; \frac{p \mu-r}{b}-\frac{\sigma \delta}{D(\sigma-1)}+1
$$

Next, we consider the case in which $s=s_{m}$ and $1 \leq s \leq m-1$. From equation (6) we have

$$
\begin{aligned}
& J(s, m)-J(s, m-1)= \\
& =-K_{m}\left\{p \mu \alpha+r\left[1 \sigma+2 \sigma^{2}+\ldots+s \sigma^{s}\right]+r s\left[\sigma^{s+1}+\sigma^{s+2}+\ldots+\sigma^{m} \delta\right]\right. \\
& \left.+h\left[s \alpha+(s-1) \sigma+\ldots+1 \sigma^{s-1}\right]+b\left[1 \sigma^{s+1}+2 \sigma^{s+2}+\ldots+(m-s) \sigma^{m} \delta\right]\right\} \\
& +K_{m-1}\left\{p \mu \alpha+r\left[1 \sigma+2 \sigma^{2}+\ldots+s \sigma^{s}\right]+r s\left[\sigma^{s+1}+\sigma^{s+2}+\ldots+\sigma^{m-1} \delta\right]\right. \\
& \left.+h\left[s \alpha+(s-1) \sigma+\ldots+1 \sigma^{s-1}\right]+b\left[1 \sigma^{s+1}+2 \sigma^{s+2}+\ldots+(m-s-1) \sigma^{m-1} \delta\right]\right\} .
\end{aligned}
$$

From equation (5) of Lemma 1, after a little algebra, we obtain

$$
J(s, m)-J(s, m-1)=K_{m-1} \sigma^{m-1}\{[p \mu-J(s, m)-r s-b(m-s-1)] D(\sigma-1)-b \sigma \delta\} .
$$

The above expression is nonnegative, since $J(s, m) \geq J(s, m-1)$, and the term $J(s, m)$ inside the brackets is positive. From these observations, after a little algebra, we obtain

$$
m<\frac{p \mu+(b-r) s}{b}-\frac{\sigma \delta}{D(\sigma-1)}+1
$$

Since $(b-r) s$ is maximized at $s=0$ or at $s=S$, depending on the sign of $(b-r)$, the above implies that

$$
m<M_{1}=;^{\Delta} \frac{p \mu+\max [(b-r) S, 0]}{b}-\frac{\sigma \delta}{D(\sigma-1)}+1 .
$$

Note that the bound $M_{1}$ is greater than $M_{0}$.

Finally, we assume that $s_{m}=m$ and compare $J(m, m)$ and $J(m-1, m-1)$. From 
equations (6) and (5) we obtain

$$
\begin{aligned}
& J(m, m)-J(m-1, m-1)= \\
& =-K_{m}\left\{p \mu \alpha+r\left[1 \sigma+2 \sigma^{2}+\ldots+m \sigma^{m} \delta\right]+h\left[m \alpha+(m-1) \sigma+\ldots+1 \sigma^{m-1}\right]\right\} \\
& \quad+K_{m-1}\left\{p \mu \alpha+r\left[1 \sigma+2 \sigma^{2}+\ldots+(m-1) \sigma^{m-1} \delta\right]\right. \\
& \left.\quad+h\left[(m-1) \alpha+(m-2) \sigma+\ldots+1 \sigma^{m-2}\right]\right\} . \\
& \quad K_{m-1} \sigma^{m-1}\{[p \mu-J(m, m)-r(m-1)] D(\sigma-1)-r \sigma \delta\}-K_{m-1} h\left(\alpha+\sigma+\ldots+\sigma^{m-1}\right) . \\
& =-h+K_{m-1} \sigma^{m-1}\{[p \mu-J(m, m)-r(m-1)+h / \sigma] D(\sigma-1)-r \sigma \delta\} .
\end{aligned}
$$

As in the previous cases, the inequalities $J(m, m)>0$ and $J(m, m) \geq J(m-1, m-1)$ imply that $[p \mu-r(m-1)+h / \sigma] D(\sigma-1)-r \sigma \delta>0$. This condition yields

$$
m<M_{2}=; \frac{p \mu-h / \sigma}{r}-\frac{\sigma \delta}{D(\sigma-1)}+1 .
$$

Finally, if $\sigma=1$, then it can be shown that the bounds (C2) and (C3) are still valid if we replace $D(\sigma-1)$ by 1 . Therefore, $m$ is bounded from above by $M=\max \left\{M_{1}, M_{2}\right\}$.

\section{References}

Buzacott, J.A., and Shanthikumar, J.G., 1993. Stochastic Models of Manufacturing Systems. Prentice Hall, Englewood Cliffs, NJ.

Caldentey, R.A., 2001. Analyzing the make-to stock queue in the supply chain and ebusiness settings. Ph.D. dissertation, Sloan School of Management, MIT, Massachusetts.

Framinan, J.M. González, P.L., and Ruiz-Uzano, R., 2003. The CONWIP production control system: review and research issues. Production Planning \& Control, 14, 
$255-265$.

Ha, A.Y., 1997a. Inventory rationing in a make-to-stock production system with several demand classes and lost sales. Management Science, 43, 1093-1103.

Ha, A.Y., 1997b. Stock-rationing policy for a make-to-stock production system with two priority classes and backordering. Naval Research Logistics, 44, 457-472.

Hadley, G., and Whitin, T.M., 1963. Analysis of Inventory Systems. Prentice Hall, Englewood Cliffs, NJ.

Iglehart, D.L., 1963. Optimality of $(s, S)$ policies in the infinite horizon inventory problem. Management Science, 9, 259-267.

Ioannidis, S., Kouikoglou, V.S., and Phillis, Y.A., 2004. Coordinating quality, production, and sales in manufacturing systems. International Journal of Production Research, 42, 3947-3956.

Kouikoglou, V.S., and Phillis, Y.A., 2002. Design of product specifications and control policies in a single-stage production system. IIE Transactions, 34, 590-600.

Kouvatsos, D.D., 1986. A maximum entropy queue length distribution for a G/G/1 finite capacity queue. ACM SIGMETRICS Performance Evaluation Review, 14, 224-236.

Moinzadeh, K., 1989. Operating characteristics of the $(S-1, S)$ inventory system with partial backorders and constant resupply times. Management Science, 35, 472-477.

Porteus, E.L., 1990. Stochastic inventory theory. Handbooks in OR \& MS, Vol. 2, D.P. Heyman and M.J. Sobel (Eds.), 605-652, North-Holland.

Sethi, S.P., and Cheng, F., 1997. Optimality of $(s, S)$ policies in inventory models with markovian demand. Operations Research, 45, 931-939. 
Song, D.P., 2006. Optimal production and backordering policy in failure-prone manufacturing systems. IEEE Transactions on Automatic Control, 51, 906-911.

Spearman, M. L., Woodruff, D. L., and Hopp, W. J., 1990. Conwip: a pull alternative to Kanban. International Journal of Production Research, 28, 879-894.

Spearman, M. L., and Zazanis, M. A., 1992. Push and pull production systems: issues and comparisons. Operations Research, 40, 521-532.

Stidham, Jr., S., 1985. Optimal control of admission to a queueing system. IEEE Transactions on Automatic Control, AC-30, 705-713.

Zipkin, P.H., 2000. Foundations of Inventory Management. McGraw-Hill, New York, NY. 
List of Tables and Figures

http://mc.manuscriptcentral.com/tprs Email: ijpr@lboro.ac.uk 


\begin{tabular}{|c|c|c|c|c|}
\hline \multicolumn{3}{|c|}{ Production system } & Queueing system & \multirow[t]{2}{*}{ Remarks } \\
\hline$n_{r}$ & $n_{h}$ & $n_{b}$ & $n$ & \\
\hline 0 & $s$ & 0 & 0 & \\
\hline 1 & $s-1$ & 0 & 1 & rows valid only when $s>0$ \\
\hline$\ldots$ & $\ldots$ & $\ldots$ & $\ldots$ & \\
\hline$s$ & 0 & 0 & $s$ & \\
\hline $\max \{s, 1\}$ & 0 & 1 & $s+1$ & rows valid only when \\
\hline $\begin{array}{c}\cdots \\
\max \{s, 1\}\end{array}$ & $\ddot{0}$ & $\begin{array}{c}\cdots \\
m-s\end{array}$ & $\begin{array}{l}\cdots \\
m\end{array}$ & $c=m-s>0$ \\
\hline
\end{tabular}

Table 1. Relationship between the production system and the queueing system

\begin{tabular}{c||cccc|cccc|cccc}
\hline \hline \multicolumn{1}{c||}{} & \multicolumn{10}{c|}{ Demand Rate } \\
\cline { 2 - 13 } & \multicolumn{1}{c|}{$\lambda=3<<\mu$} & \multicolumn{1}{c|}{$\lambda=10=\mu$} & \multicolumn{4}{c}{$\lambda=30>\mu$} \\
\hline \hline Policy & $s$ & $c$ & $q$ & $J$ & $s$ & $c$ & $q$ & $J$ & $s$ & $c$ & $q$ & $J$ \\
\hline BB & 0 & 13 & - & 26.36 & 1 & 4 & - & 70.00 & 1 & 2 & - & 84.62 \\
LS & 2 & 0 & 0.00 & 18.06 & 3 & 0 & 0 & 60.00 & 3 & 0 & 0.00 & 82.50 \\
CB & 0 & $\infty$ & 1.00 & 26.36 & - & - & - & $-\infty$ & - & - & - & $-\infty$ \\
RAC & 0 & $\infty$ & 1.00 & 26.36 & 1 & $\infty$ & 0.72 & 63.08 & 3 & $\infty$ & 0.07 & 82.52 \\
MTO/BB & 0 & 13 & - & 26.36 & 0 & 5 & - & 66.67 & 0 & 3 & - & 79.88 \\
MTO/RAC & 0 & $\infty$ & 1.00 & 26.36 & 0 & $\infty$ & 0.78 & 56.37 & 0 & $\infty$ & 0.25 & 56.37 \\
\hline \hline
\end{tabular}

Table 2. Control parameters and profit rate for an $M / M / 1 / m$ system for various demand 


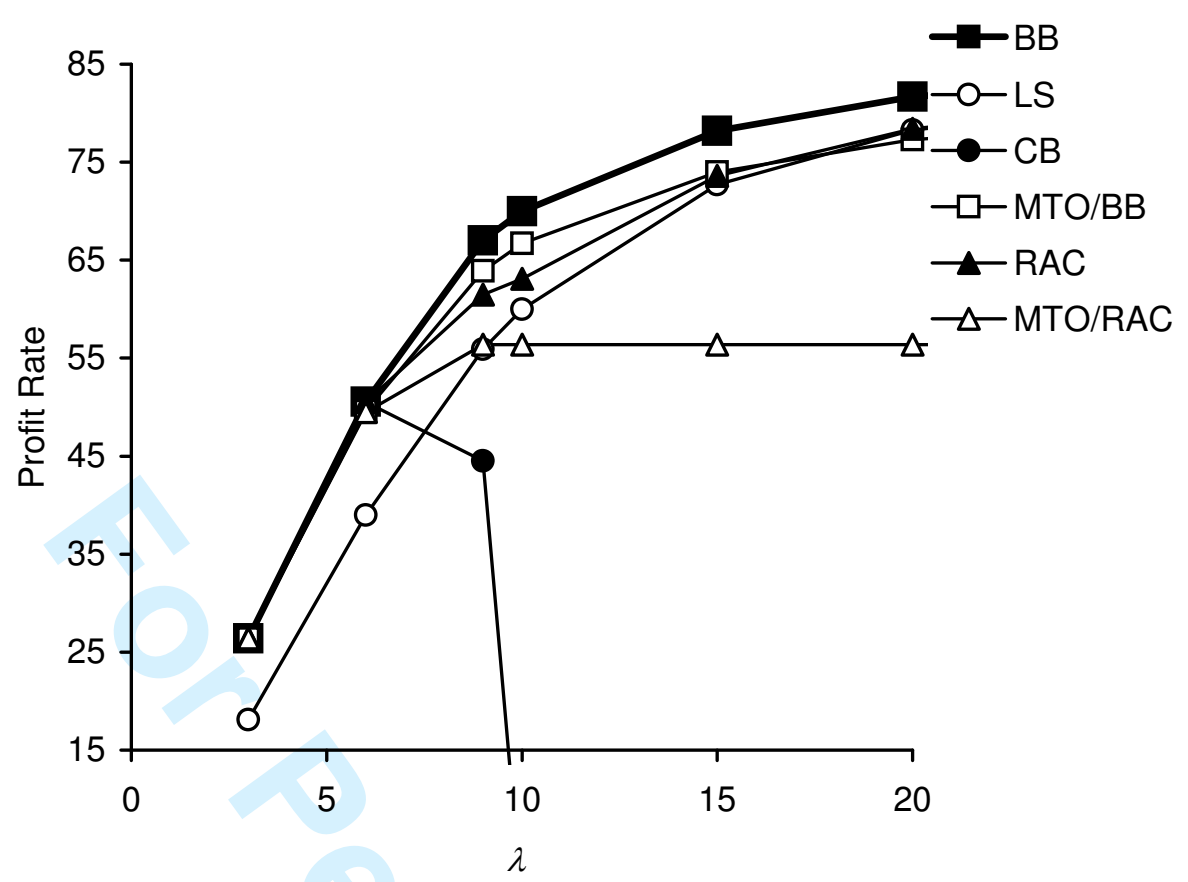

Figure 1. Mean profit rate versus $\lambda$ for an $M / M / 1 / m$ system

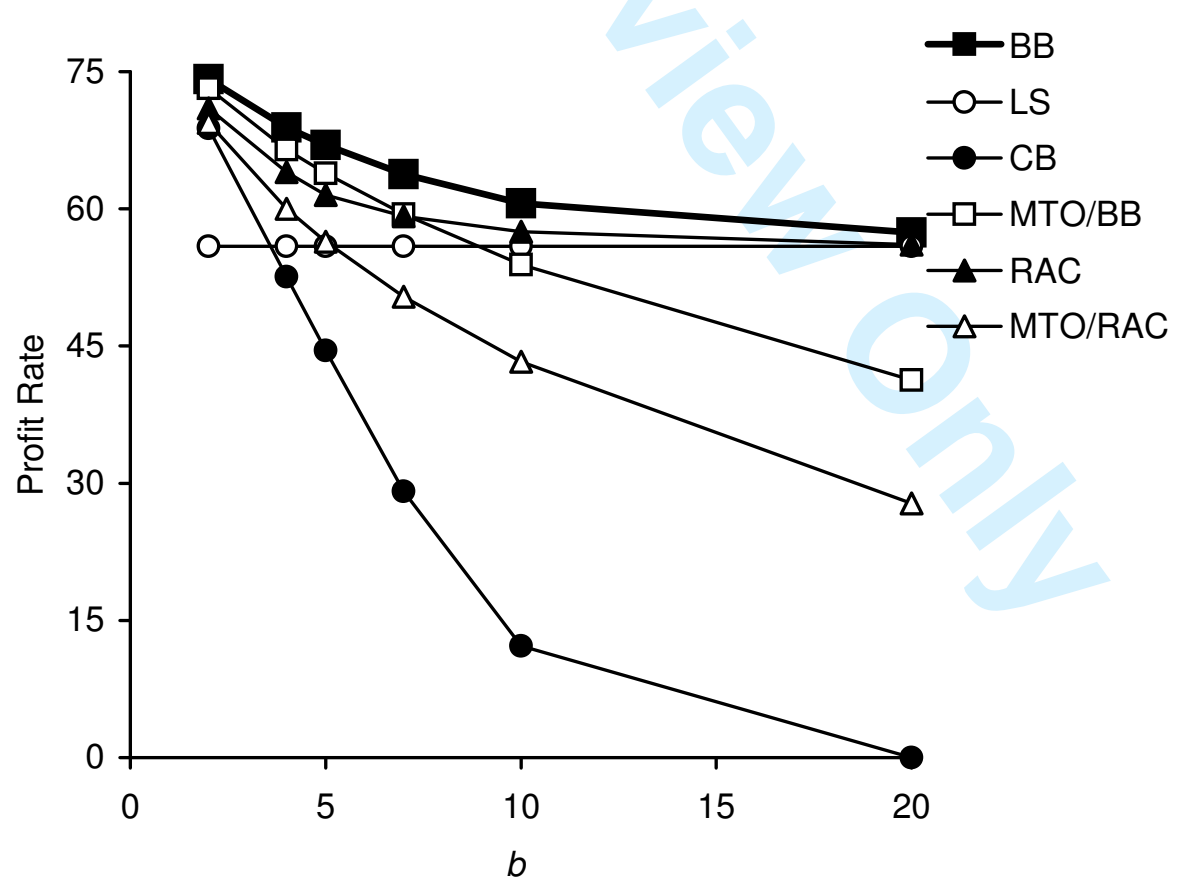

Figure 2. Mean profit rate versus $b$ for an $M / M / 1 / m$ system 


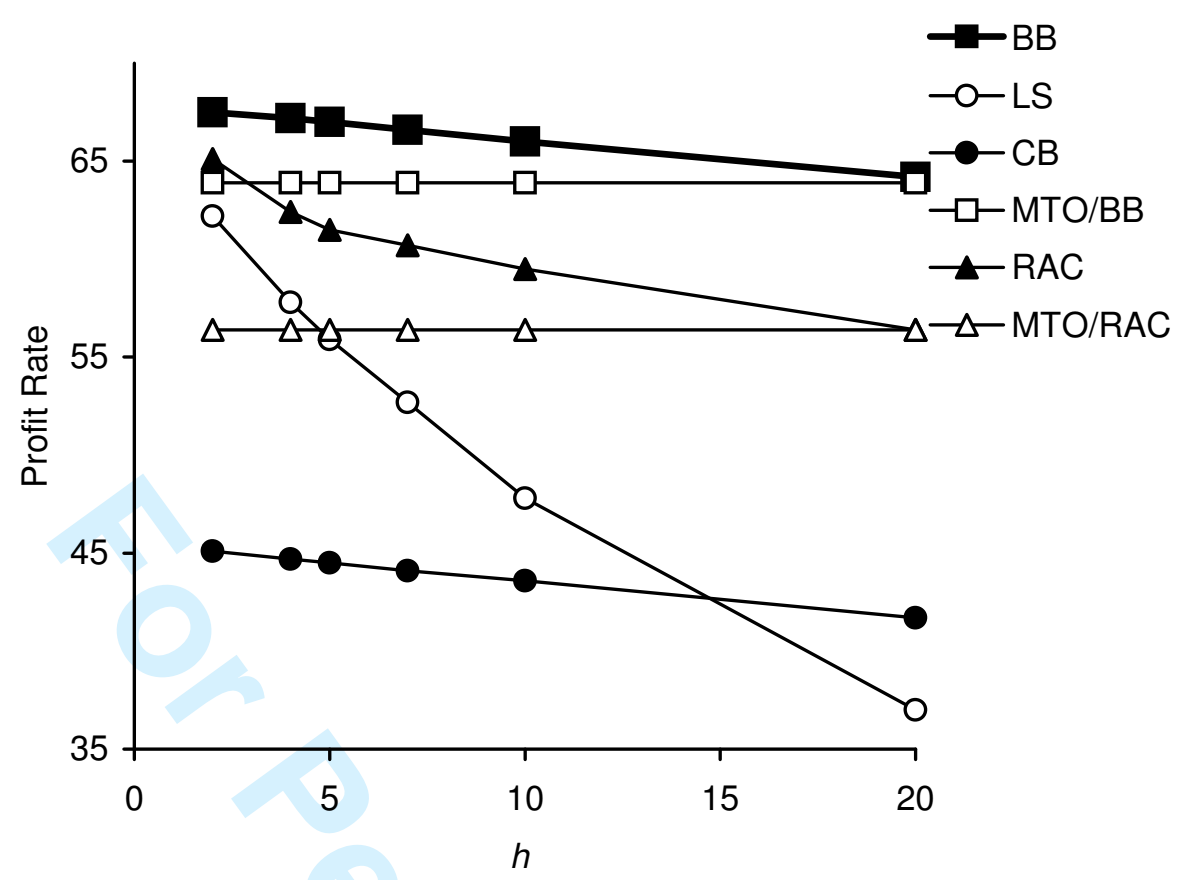

Figure 3. Mean profit rate versus $h$ for an $M / M / 1 / m$ system

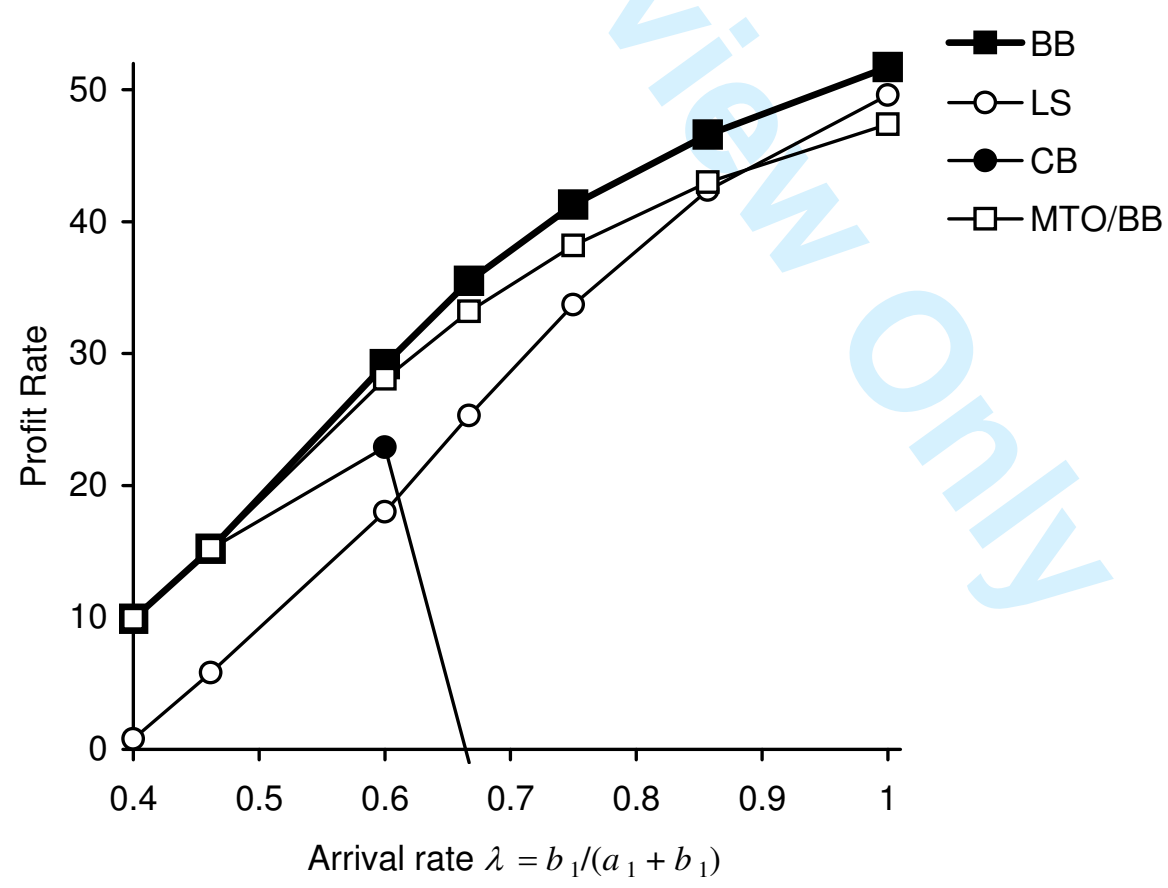

Figure 4. Mean profit rate versus $\lambda$ for Model II 


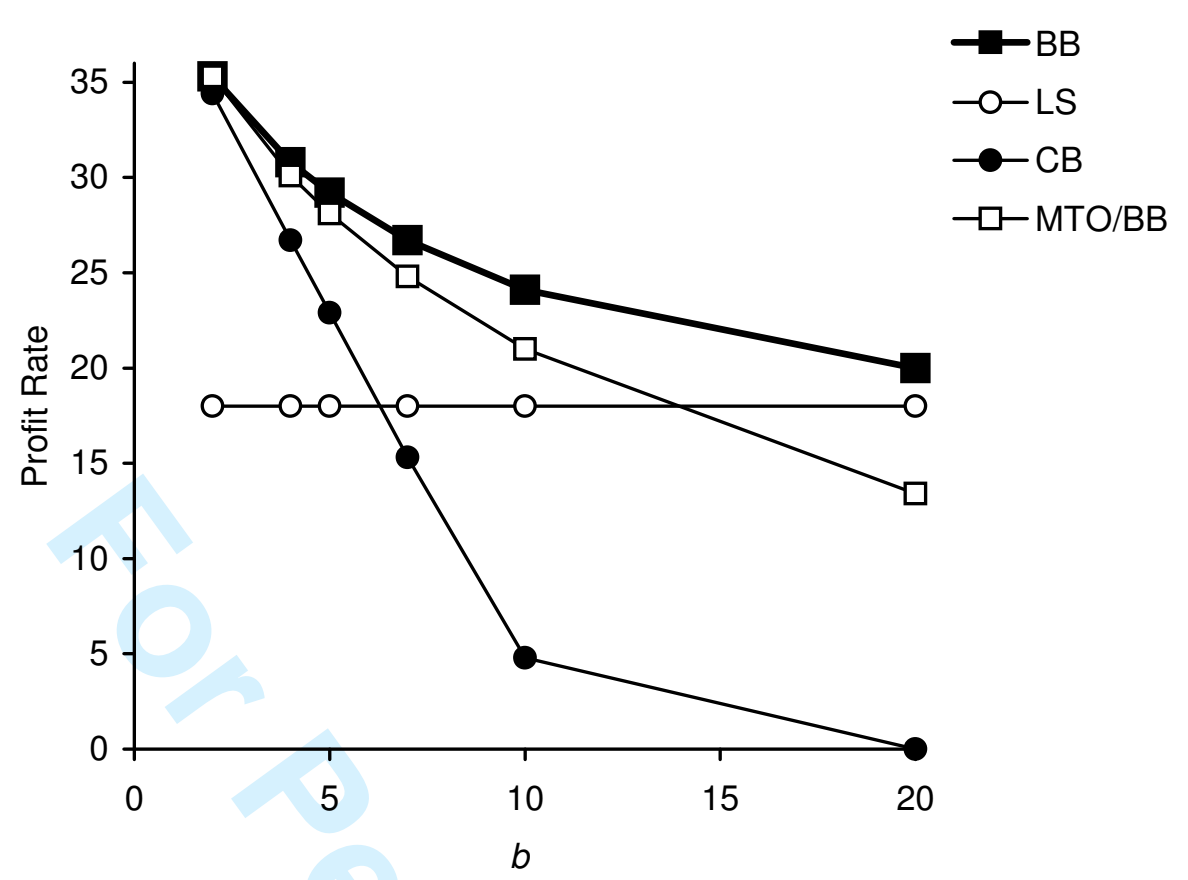

Figure 5. Mean profit rate versus $b$ for Model II

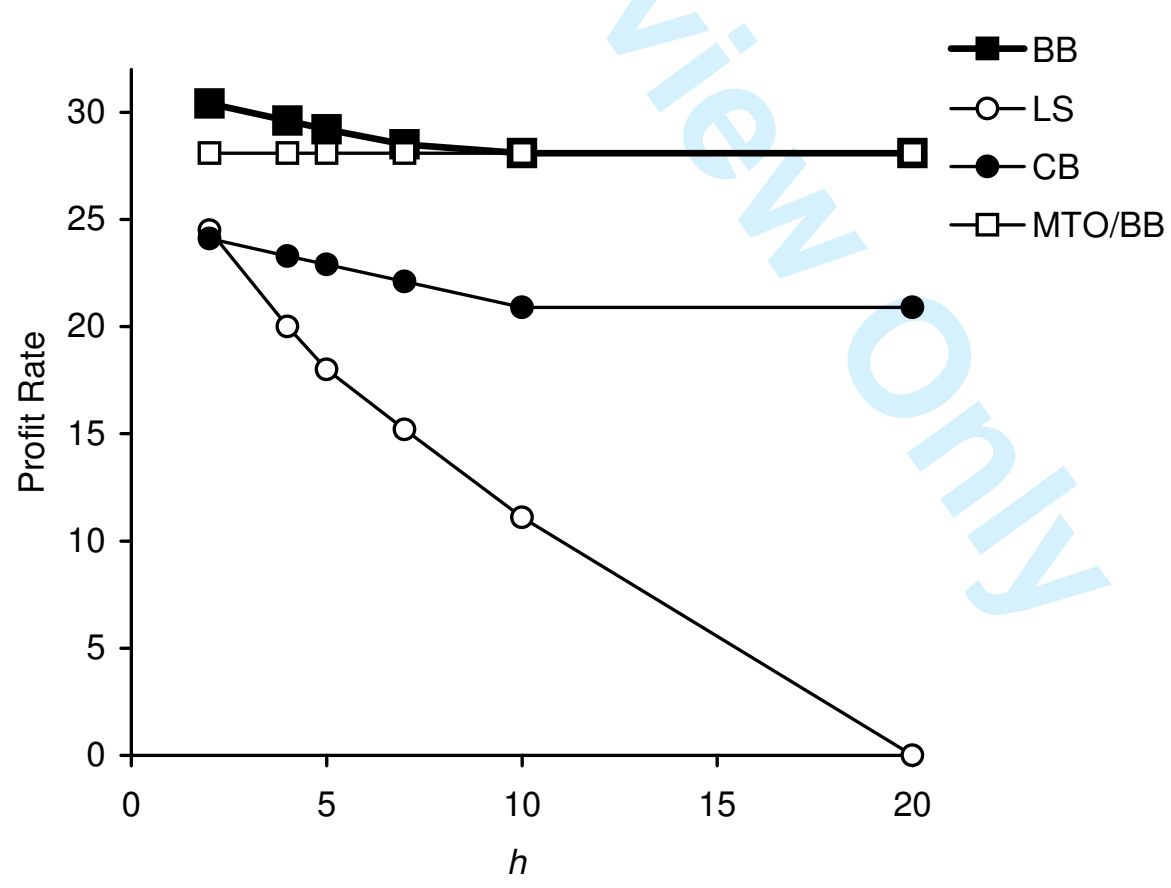

Figure 6. Mean profit rate versus $h$ for Model II 